OPEN ACCESS

Edited by:

Mariella Pazzaglia,

Sapienza University of Rome, Italy

Reviewed by:

Annalisa Setti,

University College Cork, Ireland

Masoud Sadeghi,

Kermanshah University of Medical

Sciences, Iran

*Correspondence:

Sulin Zhang

156933024@qq.com

Weijia Kong

entwjkong@hust.edu.cn

†These authors have contributed equally to this work

Specialty section:

This article was submitted to

Perception Science,

a section of the journal

Frontiers in Neuroscience

Received: 23 November 2019

Accepted: 06 April 2020

Published: 28 April 2020

Citation:

Zhang S, Xu W, Zhu Y, Tian E and Kong W (2020) Impaired Multisensory

Integration Predisposes the Elderly

People to Fall: A Systematic Review.

Front. Neurosci. 14:411.

doi: 10.3389/fnins.2020.00411

\section{Impaired Multisensory Integration Predisposes the Elderly People to Fall: A Systematic Review}

\author{
Sulin Zhang ${ }^{1,2 *}$, Wenchao $\mathrm{Xu}^{1+}$, Yuting Zhu ${ }^{1}$, E. Tian ${ }^{1}$ and Weijia Kong ${ }^{1,2,3 *}$ \\ ${ }^{1}$ Department of Otorhinolaryngology, Union Hospital, Tongji Medical College, Huazhong University of Science and \\ Technology, Wuhan, China, ${ }^{2}$ Institute of Otorhinolaryngology, Union Hospital, Tongji Medical College, Huazhong University of \\ Science and Technology, Wuhan, China, ${ }^{3}$ Key Laboratory of Neurological Disorders of Education Ministry, Tongji Medical \\ College, Huazhong University of Science and Technology, Wuhan, China
}

Background: This systematic review pooled all the latest data and reviewed all the relevant studies to look into the effect of multisensory integration on the balance function in the elderly.

Methods: PubMed, Web of Science and Scopus were searched to find eligible studies published prior to May 2019. The studies were limited to those published in Chinese and English language. The quality of the included studies was assessed against the Newcastle-Ottawa Scale or an 11-item checklist, as recommended by Agency for Healthcare Research and Quality (AHRQ). Any disagreement among reviewers was resolved by comparing notes and reaching a consensus.

Results: Eight hundred thirty-nine records were identified and 17 of them were included for systematic review. The result supported our assumption that multisensory integration works on balance function in the elderly. All the 17 studies were believed to be of high or moderate quality.

Conclusions: The systematic review found that the impairment of multisensory integration could predispose elderly people to fall. Accurate assessment of multisensory integration can help the elderly identify the impaired balance function and minimize the risk of fall. And our results provide a new basis for further understanding of balance maintenance mechanism. Further research is warranted to explore the change in brain areas related to multisensory integration in the elderly.

Keywords: multisensory integration, balance, older adults, systematic review, neurophysiology

\section{INTRODUCTION}

\section{Rationales}

Epidemiological studies showed that elderly people aged 60 or above are expected to account for $20 \%$ of overall population by 2050 (World Health Organization, 2015; United Nations, 2017). Sufficient mobility, i e., the ability to move physically and engage in daily activities (Lowry et al., 2012) is essential for normal living. Such movement and activities requires maintenance of physical balance (Stel et al., 2003). Moreover, each year, 30\% of community-dwelling elderly people aged 65 and above suffer from falls (Blake et al., 1988) and the rate rose to $32-42 \%$ in those aged over 70 (Stalenhoef et al., 2002). The rate of fall-related hospitalization in people at the age of 60 and above 
was somewhere between 1.6 and 8.9 per 10,000 people (World Health Organization, 2007) and fall-related fatalities reached up to $40 \%$ of all injury-related deaths (Rubenstein, 2006). These data show that the balance function is closely associated with the quality of life of the elderly. Mechanistically, balance is achieved or maintained by the integrated input of vision, vestibular and somatic sensation into the central nervous system, and the following responses of the musculoskeletal system (Katsarkas, 1994). It has been substantiated that uni-sensory acuity degrades as a result of aging (Chisolm et al., 2003; Stein and Stanford, 2008), and, presumably, such impaired acuity predisposes elderly people to imbalance and falls (Tromp et al., 2001). Furthermore, elderly people tend to suffer from multiple diseases, including physical and mental disorders, that may well-cause imbalance or falls.

Nonetheless, high sensory acuity is not the only prerequisite for maintaining balance. Balance maintenance may also depend on efficient multisensory integration in the brain. Multisensory integration refers to the process by which the nervous system integrates information from different perceiving processes, such as sight (Wassenhove et al., 2005), hearing (Peelle and Sommers, 2015; Morís Fernández et al., 2017), and other somatosensory events (Foxe et al., 2000), into a unified, coherent, and stable single multisensory process (Bolognini et al., 2015; Talsma, 2015). Furthermore, multisensory integration can compensate for the inadequacy of information from a single sense (Schroeder and Foxe, 2005; Diaconescu et al., 2013; Bizley et al., 2016), provide redundant information that brings about faster detection responses (Kinchla, 1974) and help perceive as much as meaningful information in the environment (Spence, 2011). Such integration has been a subject of active studies on human learning (Seitz et al., 2006), memory (Barutchu et al., 2019), and consciousness (Price, 1999). So far, the physiological and pathological roles of multisensory integration have not been fully understood. However, many studies have shown that, with aging, the multisensory integration is progressively impaired. Some studies showed that multisensory integration is enhanced among the elderly compared to young adults (Laurienti et al., 2006; DeLoss et al., 2013; Ramkhalawansingh et al., 2016) while other researches showed the otherwise (Stephen et al., 2010).

\section{Objectives}

Few studies examined the effect of multisensory integration on the balance function of elderly people (Allison et al., 2006; Bronstein, 2016) and so far, no consistent conclusions have been reached yet. In this systematic review, we pooled all the latest data and comprehensively analyzed the relevant studies to further look into the effect of multisensory integration on balance in the elderly.

\section{Questions to Be Answered}

This study tried to answer the following questions: Does multisensory integration work on balance function in elderly people? Which neurophysiological theories or hypotheses can explain their association?

\section{METHODS}

\section{Study Design}

Systematic review.

\section{Participants, Interventions, Comparators}

Participants: elderly people. Interventions: normal multisensory integration. Comparators: impaired multisensory integration. Outcome: balance function.

\section{Protocol of the Systematic Review Protocol}

The protocol for this systematic review was registered with the international PROSPERO database and the trial registration number is CRD42019134526. Protocol details are available from http://www.crd.york.ac.uk/PROSPERO/display_record. php?ID=CRD42019134526.

\section{Search Strategy}

This review was conducted in line with the Preferred Reporting Items for Systematic Reviews and Meta-Analyses (PRISMA) statement (Moher et al., 2009) and consisted of a systematic search of the major databases, formulation of inclusion or exclusion criteria, quality assessment, and data extraction. PubMed, Web of Science (WOS), Scopus were searched to identify qualified studies published before May 2019. The studies were limited to those published in the Chinese language and English language. The following terms were employed: "*sensory integration" and "balance OR equilibrium" and "older people," where "*" was the wild card. When we searched multisensory integration, we used "'Sensory integration' OR 'multisensory integration' OR 'crossmodal integration' OR 'crossmodal integration' OR 'intersensory integration' OR 'multimodal integration' OR multisensory OR crossmodal OR cross-modal OR 'crossmodal sensory integration' OR 'cross-modal sensory integration' OR 'multisensory interaction."' When we searched balance, “"posture control' OR 'postural control' OR 'gesture control' OR 'postural balance' OR equilibrium OR balance" is our terms. And we used "geriatr* OR aged OR elderly OR gerontol* OR 'older adults' OR aging." The titles and abstracts of retrieved articles were reviewed and assessed independently by two reviewers to identify eligible studies against the inclusion or exclusion criteria and the full text of the eligible articles was retrieved for further analysis. If any disagreement occurred, a consensus was reached by further referring to the inclusion or exclusion criteria.

\section{Data Sources, Studies Sections, and Data Extraction}

This systematic review pooled the most up-to-date data on the effect of multisensory integration on balance in elderly people. A study was included if (1) the target population was elderly people aged 60 and over (United Nations, 2017); (2) the subjects without unisensory abnormality included were elderly people with fall history or the elderly people in whom assessment showed impaired balance function. 
Moreover, studies were excluded if: (1) they were case reports, reviews, letters, or editorial comments; (2) relevant data were insufficient or unavailable; (3) they had overlapping data or were animal studies.

Case control studies were assessed in terms of quality against the Newcastle-Ottawa Scale (NOS) (Wells et al., 2012), which consists of nine sub-items under the headings of the overall selection of groups, the comparability of study groups and the overall ascertainment of the exposure in all groups. For each subitem, one point was awarded. So, the highest score was nine. In line with other systematic reviews (Ma et al., 2016; de Dieuleveult et al., 2017), five points were selected as cut-off values. Studies that scored five or higher were deemed of high quality. The quality of cross-sectional studies was assessed using an 11-item checklist which was recommended by Agency for Healthcare Research and Quality (AHRQ). An item would be rated " 0 " if its answer was "NO" or "UNCLEAR;" if the answer was "YES," "1" point was awarded. Study quality was assessed on an 0-11 scale: low quality $=0-3$, moderate quality $=4-7$, high quality
$=8-11$. Two investigators independently assessed the quality of each eligible study. Any disagreement was resolved by reaching a consensus.

By examining the abstract, introduction, methods, and results of each individual study, the features of the studies were identified and listed in a table, including sample size, age range, gender, materials and methods, and results related to the effect of multisensory integration. Data were extracted by using a predesigned form. Two reviewers separately extracted the data as per criteria. Any disagreement was settled by reviewing the full text of the paper involved.

\section{Data Analysis}

The tests used in these studies were too heterogeneous to be pooled. Even though some studies had used the same tests, the test conditions and the measurements varied substantially. In view of these, we only provided a qualitative synthesis of the results.

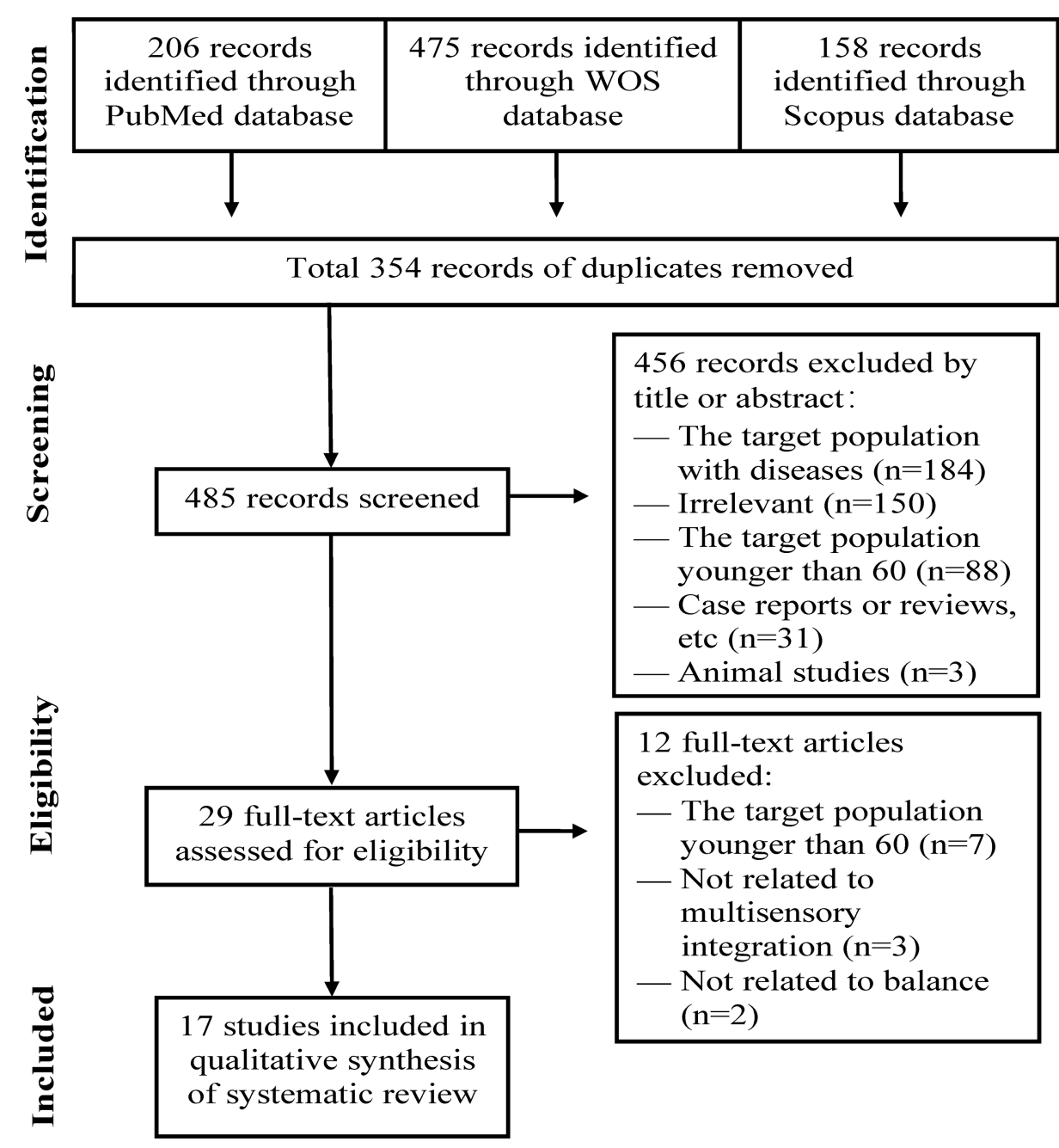

FIGURE 1 | PRISMA flow diagram of study selection process. 


\section{RESULTS}

The initial database search identified 839 records that were potentially eligible for inclusion in the systematic review. Then, 354 duplicate records were eliminated. Future review of the title and abstract removed 456 records that did not satisfy the inclusion criteria. Upon assessment of the remaining 29 full-text articles, 12 more articles were ruled out. As a result, 17 studies were eventually included for qualitative synthesis of systematic review (Figure 1).

\section{Study Selection and Characteristics}

In these studies, 14 studies were case control studies and three were cross-sectional studies. Against NOS (Table 1), all the 14 studies scored high points on quality, i.e., five or higher and were considered to have strong power. The quality assessment of crosssectional studies is presented in Table 2. Three studies were of moderate quality.

In terms of the inclusion or omission of a group of the fallprone elderly people, all the studies were divided into two groups: studies involving fall-prone elderly people $(n=6)$ and those without including fall-prone elderly people $(n=11)$. The sample size and age range of the 17 studies are summarized in Table 3.
In some studies, other factors were also controlled to ensure the comparability between groups, including gender, education background, intelligence quotient (IQ), among others.

\section{Synthesized Findings}

The eligible studies were evaluated in terms of participants, study design, and findings related to the effect of multisensory integration. The results of the evaluation are presented in Tables 4, 5 .

\section{Studies Containing Fall-Prone Elderly People}

Studies in this group examined the sway variability when subjects performed experimental tasks in various multisensory integration situations. Participants were required to maintain posture whenever possible in different test situations. First of

TABLE 2 | Quality assessment of cross-sectional studies.

Study Rosengren et al. (2007) Palazzo et al. (2015) Sparto et al. (2018)

Scores 6

7 7

TABLE 1 | Quality assessment with the Newcastle-Ottawa Quality Assessment Scale (NOS).

\begin{tabular}{|c|c|c|c|c|c|c|c|c|c|c|}
\hline \multirow[t]{2}{*}{ References } & \multicolumn{4}{|c|}{ Selection $(\max =4)$} & \multicolumn{2}{|c|}{ Comparability $(\max =2)$} & \multicolumn{3}{|c|}{ Exposure $(\max =3)$} & \multirow{2}{*}{$\begin{array}{c}\text { Quality } \\
\text { score } \\
(\max =9)\end{array}$} \\
\hline & $\begin{array}{l}\text { Is the } \\
\text { population } \\
\text { definition } \\
\text { adequate? }\end{array}$ & $\begin{array}{c}\text { Representative } \\
\text { ness of the } \\
\text { population }\end{array}$ & $\begin{array}{l}\text {-Selection of } \\
\text { controls }\end{array}$ & $\begin{array}{l}\text { Definition of } \\
\text { controls }\end{array}$ & $\begin{array}{c}\text { Study } \\
\text { controls for } \\
\text { MSI }\end{array}$ & $\begin{array}{c}\text { Study } \\
\text { controls for } \\
\text { additional } \\
\text { factor }\end{array}$ & $\begin{array}{l}\text { Ascertainment } \\
\text { of exposure }\end{array}$ & $\begin{array}{l}\text { Same } \\
\text { method of } \\
\text { ascertainment } \\
\text { for cases } \\
\text { and controls }\end{array}$ & $\begin{array}{l}\text { Non- } \\
\text { response } \\
\text { rate }\end{array}$ & \\
\hline $\begin{array}{l}\text { Redfern et al. } \\
\text { (2001) }\end{array}$ & * & * & - & * & * & * & * & * & * & 8 \\
\hline Allison et al. (2006) & * & * & * & * & * & - & $\star$ & - & * & 7 \\
\hline Jeka et al. (2006) & * & * & - & * & * & - & * & * & * & 7 \\
\hline $\begin{array}{l}\text { Redfern et al. } \\
\text { (2009) }\end{array}$ & * & * & - & * & * & * & * & * & * & 8 \\
\hline Jeka et al. (2010) & * & * & - & * & * & - & * & * & * & 7 \\
\hline Setti et al. (2011) & * & * & * & * & * & * & * & * & * & 9 \\
\hline Zhou et al. (2013) & * & * & - & * & * & - & * & * & * & 7 \\
\hline $\begin{array}{l}\text { Mahoney et al. } \\
(2014)\end{array}$ & * & * & - & * & * & - & * & * & * & 7 \\
\hline $\begin{array}{l}\text { Stapleton et al. } \\
\text { (2014) }\end{array}$ & * & * & - & * & * & - & * & * & * & 7 \\
\hline Ross et al. (2016) & * & - & - & - & * & - & * & * & * & 5 \\
\hline $\begin{array}{l}\text { Teramoto et al. } \\
(2017)\end{array}$ & * & * & - & * & * & * & * & * & * & 8 \\
\hline $\begin{array}{l}\text { Mahoney et al. } \\
\text { (2018) }\end{array}$ & * & * & - & * & * & * & * & * & * & 8 \\
\hline Anson et al. (2019) & * & * & - & * & * & * & * & * & * & 8 \\
\hline $\begin{array}{l}\text { Redfern et al. } \\
\text { (2019) }\end{array}$ & * & * & - & * & * & * & * & * & * & 8 \\
\hline
\end{tabular}

(*), Acceptable; (-), Not acceptable; MSI, multisensory integration. 
TABLE 3 | Sample size and age range of the two groups.

\begin{tabular}{lccccc}
\hline Groups & \multicolumn{2}{c}{ Sample size (range, mean) } & & \multicolumn{2}{c}{ Age (years) } \\
\cline { 2 - 3 } \cline { 5 - 6 } & $\begin{array}{c}\text { Healthy } \\
\text { older adults }\end{array}$ & $\begin{array}{c}\text { Fall-prone } \\
\text { older adults }\end{array}$ & & $\begin{array}{c}\text { Healthy } \\
\text { older adults }\end{array}$ & $\begin{array}{c}\text { Fall-prone } \\
\text { older adults }\end{array}$ \\
\hline $\begin{array}{l}\text { Studies with fall-prone } \\
\text { older adults }\end{array}$ & $7-50,22$ & $15-94,32$ & & $60-93$ & $60-92$ \\
$\begin{array}{l}\text { Studies without } \\
\text { fall-prone older adults }\end{array}$ & $15-376,103$ & - & $60-88$ & - \\
\hline
\end{tabular}

$(-)$, not reported.

all, in some studies participants were asked to perform a soundinduced flash illusion task and were exposed to consistent or inconsistent audio-visual stimuli. The subjects were required to record the number of visual or auditory stimuli (Setti et al., 2011; Stapleton et al., 2014). Allison et al. (2006) investigated sway variability of subjects who were asked to look at moving visual stimuli in a moving room and to touch a moving touch plate. Other researchers used the visual display oscillating at different amplitudes, changing from high to low or from low to high amplitudes (Jeka et al., 2006, 2010). And Zhou et al. (2013) employed modified clinical tests for multisensory integration, with subjects standing on a firm or foam surface with eyes open or closed.

All these studies yielded interesting findings. First of all, Setti et al. (2011) and Stapleton et al. (2014) suggested that fallprone elderly people experienced greater number of illusions than their healthy counterparts, especially under incongruent condition. Inefficient audiovisual integration might predispose the fall-prone elderly people to illusion. So, balance maintenance and the related incidence of falls are believed to be associated with a general impairment in the multisensory integration.

Second, Jeka et al. $(2006,2010)$ illustrated, in two studies, that poor balance control in the fall-prone elderly people was related to their inability to properly re-weight multisensory inputs. The ability to re-weight sensory inputs is important for postural control in elderly people. Central sensory re-weighting processes are believed to deteriorate with age and are inefficient in fall-prone adults. However, another study, by Allison et al. (2006), didn't support the hypothesis that the multisensory reweighting process is impaired in fall-prone elderly people compared to their healthy counterparts. Nonetheless, they found that postural variability tended to increase when sensory stimulus amplitude or moving speeds increased. The discrepancy among the three studies might be ascribed to the difference in their trial duration, because longer time was needed to reflect actual re-weighting impairment.

Finally, by using a simple technique, Zhou et al. (2013) demonstrated that fall-prone elderly adults tended to have a significantly shorter stance time in modified clinical balance tests for multisensory integration, when standing on a firm or foam surface with eyes open or closed.

\section{Studies Containing no Fall-Prone Elderly People}

Studies in this group investigated participants' ability to maintain balance. Participants were required to achieve balance whenever possible. Some authors examined the postural sway when participants performed modified clinical tests, standing on various surfaces with eyes open or closed (Redfern et al., 2001, 2019; Rosengren et al., 2007; Palazzo et al., 2015; Sparto et al., 2018; Anson et al., 2019) in the light or in the dark (Redfern et al., 2009). Similarly, in some studies, participants were asked to stand on a force platform with eyes open or closed under quiet or noisy condition (Ross et al., 2016). Moreover, subjects were examined for reaction time when they were instructed to quickly react to all stimuli given on one hemispace (left or right), regardless of spatial distance (near/middle/far), and target modality (visual/tactile/visuotactile). And to assess the sensorimotor function of participants, postural stability tests, and the Timed Up and Go (TUG) were used (Teramoto et al., 2017). Some researchers recorded uni-pedal stance time of subjects with different MSI (multisensory integration) (Mahoney et al., 2014, 2018).

Of the 11 eligible studies, eight comparable researches yielded similar results. They found that postural sway increased significantly when balance tasks became progressively more complex, suggesting that the multisensory integration was important in the balance maintenance, and postural control in the upright position. Normal multisensory integration was found to decrease the balance-related incidents, such as falls in elderly people. In three of the eight studies, subjects were asked to perform inhibition tasks and their results revealed that perceptual inhibition might be a component of multisensory integration process (Redfern et al., 2001, 2009, 2019). The ability to isolate and process appropriate sensory stimuli whilst inhibiting irrelevant stimuli is essential for achieving behavioral goals. Teramoto et al. (2017) illustrated that multisensory integration was enhanced, especially in practically all elderly people with poor postural stability. Another two studies (Mahoney et al., 2014, 2018) found significant difference in uni-pedal stance time among subjects with different MSI. Excellent and good integrators had the longest uni-pedal stance time while poor and deficient integrators had the shortest one.

\section{DISCUSSION}

\section{Main Findings}

To understand the effect of multisensory integration on balance function in elderly people, in this study, we searched major databases for eligible articles, conducted a systematic review and found that the multisensory integration and balance were intimately related in elderly people.

Balance in elderly adults is multifaceted and is maintained by the integration of vision, vestibular sensation, and somatic sensation inputs into the central nervous system (CNS), and ensuing responses of the musculoskeletal system (Katsarkas, 1994). Any change in these signal inputs can lead to impairment of balance function. Normally, these functions gradually deteriorate with age (Teasdale et al., 1991). Though previously a great many studies looked into the vestibular dysfunction since it is highly prevalent, few researchers examined the balance in the healthy elderly people (Bronstein, 2016). Therefore, in this study, we focused on the balance function in healthy elderly people, with an attempt to understand the effect of multisensory integration 
Tests

Results related to the effect of

multisensory integration

\section{Conditions}

\section{Studies with fall-prone elderly people group $(n=6)$}

Jeka et al. (2006) Healthy older adults: 7; 79-84; 81.1(2.12); n.r; fall-prone older adults: $15 ; 68-84 ; 80.7(5.47) ; n . r ;$ young adults: 12; 18-27; 22.0(3.12); n.r

Postural task

Jeka et al. (2010)

Healthy older adullts: 25; 70-93; 76.6(5.6):44\%; fall-prone older adults: $17 ; 72-92 ; 79.9(7.3) ; 40 \%$; young adults: $21 ; 20-30 ; 23(2.2) ; 34 \%$

Healthy older adults: 16; n.r.; n.r; 56.3\%; fall-prone older adults: 16; n.r.; n.r; 25\%; young adults: 16; n.r.; $24.4(4) ; 43.8 \%$

Zhou et al. (2013)

Healthy older adults: 50; n.r; 79.55(5.73); 48\%; fall-prone older adults: 94; n.r; 81.84(4.69); 50\%

Balance test
Healthy older adults: 21; n.r; 72.2(4.69); 57.1\%; $\begin{array}{ll}\text { Stapleton et al. (2014) } & \text { Healthy older adults: 21; n.r; 72.2(4.69); 57.1\%; } \\ & \text { fall-prone older adults: 23; n.r; 73.95(4.94); 52.2\% }\end{array}$
Allison et al. (2006) Healthy older adults: 15; n.r.; 79(3); n.r.; fall-prone older adults: 28; n.r.; 83(4); n.r; young adults: 10; 19-28; n.r. (n.r.); n.r

Postural task

Moving visual stimulus projected onto Maintain posture a screen in a moving room.

Participants held their fingertip

touching a moving touch plate. Touch

amplitude (mm): visual amplitude

(mm): (A) 8:2, (B) 4:2, (C) 2:2, (D) 2:4,

(E) 2:8

(A) Oscillating at different amplitudes; Maintain posture

(B) simultaneously oscillating at a

single amplitude and translating to the

right at different speeds: $4 \mathrm{~mm}-4$

$\mathrm{cm} / \mathrm{s}, 4 \mathrm{~mm}-1 \mathrm{~cm} / \mathrm{s}, 4 \mathrm{~mm}-0 \mathrm{~cm} / \mathrm{s}, 8$

$\mathrm{mm}-0 \mathrm{~cm} / \mathrm{s}$.

The virtual visual scene oscillated sinusoidally $0.4 \mathrm{~Hz}$. The initial amplitude was either 3 or $12 \mathrm{~mm}$. After $60 \mathrm{~s}$ the oscillation amplitude switched from 3 to $12 \mathrm{~mm}$ or vice versa, and remained at this amplitude for $120 \mathrm{~s}$

Sound-induced flash illusion (A) Visual: 1 or 2 flashes, (B) Auditory 2 beeps, (C) Audiovisual: (1) Congruent: 1 flash/ 1 beep or 2 flashes/2 beeps, (2) Illusory: Onset of beep precede or follow flash, Different SOA

Standing on (A) a firm surface with eyes open, (B) a firm surface with eyes closed, (C) a foam surface with eyes open, (D) a foam surface with eyes closed

(A) Postural task, (B) (A) Postural position: (1)sitting, (2) standing; (B) Audiovisual: (1)

Congruent: 1 flash/ 1 beep or 2

flashes/2 beeps, (2) Illusory: 1 flash/2

beeps onset of beep precede or lag

flash, Different SOA
"Our results did not support the assumption that the multisensory reweighting adaptation process is deficient in healthy and fall-prone older adults, given sufficiently intact peripheral sensation."

The four underlyingmeasures considered together showed a highly significant

Condition effect $(P<0.0001)$, a marginally significant Group effect $(P=0.052)$, and a marginally significant Group ${ }^{*}$ Condition interaction $(P=0.094)$.

"For fall-prone adults, gains continued to change over the duration of all time segments, demonstrating relatively slow adaptation and implying that the visual reweighting process in fall-prone adults is not fully achieved during the initial change in gain. Healthy older adults showed the fewest long-term changes in gain, suggesting that their initial response was often the most appropriate of the three groups."

Report the number of "Importantly, the number of illusions flashes, if no flashes, experienced by fall-prone older adults was report the number of greater than for healthy older and young greater than for healthy older and young
adults, and the number of illusions they experienced was unaffected by the onset delay between the auditory beeps from 70 to $270 \mathrm{~ms}$."

"The two groups of subjects had a statistically significant difference $(P<$ 0.05), except standing on a firm surface with eyes open and closed."

(A) Maintain posture; (B) report the number of flashes

"There was greater body sway for fall-prone older adults than healthy older adults in both mediolateral and anterior-posterior directional planes. Also, postural sway increased from the presentation of the audio-visual congruent to the audio-visual illusory conditions for the fall-prone older adults only." 
TABLE 4 | Continued

References $\quad$ Participants (sample size; age range; mean

Tests

(SD); percentage of men)
Conditions

Tasks
Redfern et al. (2001) Healthy older adults: 18; 70-85; 74(3.2); 44.4\%; Postural task young adults: 18; n.r; 22.8(1.8); 55.6\%

Rosengren et al. (2007) Healthy older adults: 20; 60-73; 65.2; 0\%

Balance test

Redfern et al. (2009) Healthy older adults: 24; 70-82; 74.2(4.4); 50\%; young adults: $24 ; 21-34 ; 25.7(3.8) ; 45.8 \%$

Postural task

(B) Inhibition task

Mahoney et al. (2014) Healthy older adults: 70; n.r; 75(6.09); 42.9\%

Palazzo et al. (2015)
Studies without fall-prone elderly people group $(n=11)$
(A) RT paradigm,

(B) Balance test

Balance test
(A) Postural task: (1) seated, (2) fixed (A) Maintain posture, floor with a stable visual environment, (B) respond as quicky (3) sway-referenced floor with a fixed as possible to the visual scene, (4) sway-referenced floor stimulus and sway-referenced visual scene; (B) information processing task: (1) none,

an auditory SRT task, (4) an inhibition reaction time task.

(A) Normal vision, fixed support; (B) eyes closed, fixed support; (C) vision swayreferenced, fixed support; (D) normal vision, support

sway-referenced; (E) eyes closed,

support surface sway-referenced; $(F)$

vision and support surface both

sway-referenced.

(A) Postural task: (1) Visual

(A) Maintain posture, conditions: (a) Eyes open in the light, (B) (1) Press a button (b) Eyes open in

on the side an arrow Sway-referenced visual scene; (2) pointed; (2) Press the Platform conditions: (a) Fixed support button on the side surface, (b) Sway-referenced floor; (B) toward the arrow Inhibition task: (1) Perceptual task: (a) pointed or on the side Congruent side arrow pointed and opposite the arrow position arrow, (b) Incongruent; (2) pointed

(A) respond to all stimuli "A one-way ANOVA revealed significant Motor task

(A) Two uni-sensory (visual and somatosensory) and one multisensory by pressing a stationary differences in mean unipedal stance time (simultaneous VS), (B) with one foot foot pedal, (B) Maintain between MSI classification [NO MSI vs. on the ground balance

bipedal stance on three different surfaces, in two different visual

conditions for each surface: open and closed eyes. young and older subjects."
(2) visual simple reaction time task, (3)
Results related to the effect of

multisensory integration
"However, older subjects' performance of toncurrent information processing task was associated with increased postural was particularly evident when both the floor and visual scene were sway referenced, which created a high degree of sensory conflict. As postural challenge was increased, RT's increased for both

"A significant main effect for condition [F $(5.95)=170, p<0.001]$ was obtained. Post-hoc analyses using Tukey HSD procedures revealed that performance on condition 1 was significantly better than that obtained in all of the other conditions.

These findings reflect typical of performance on CDP."

the older adults, perceptual inhibition as positively correlated with sway mplitude on a sway-referenced floor and with a fixed visual scene $(r=0.68, p<$ 0.001). Motor inhibition was not correlated with sway on either group. Perceptual whibition may be a component of the sensory integration process important for maintaining balance in older adults." MSI; $F(1,69)=9.51, p<0.01]$."

"The results of this study showed the importance of multisensory stimulation in postural control and in the maintenance of body balance in the orthostatic position which in turn reduced the accident risk such as falls in the elderly. sway. This increase in sway in older adults 


\section{References \\ Participants (sample size; age range; mean}

(SD); percentage of men)

Tests

\section{Conditions}

(A) Eyes closed during silence,

(B)eyes open during silence, (C)eyes

closed during noise, (D)eyes open

during noise

Teramoto et al. (2017) Healthy older adults: 20; 71-82; 74.6(2.9); n.r young adults: $11 ; 20-22 ; 21.4(0.70) ; n . r$

(A) Sensorimotor function assessment, (B) RT paradigm

Timed Up and Go (TUG) and visuotactile VTnear, VTmiddle, and VTfar)

(A) Two unisensory (visual and somatosensory) and one multisensory (simultaneous VS), (B) with one foot on the ground ostural stabilty tests, (B) tactile only, (

Results related to the effect of multisensory integration

\section{Instructions}

Maintain posture

"Standard deviation in the A-P and M-L sway and radial sway was compared across condition and using two-way analyses of variance (eyes closed vs. open analyses of variance (eyes closed vs. op
and silence vs. noise). We found main and silence vs. noise). We found main
effects ofvision $\left[F_{(1,28)}=9.36, p=0.005\right]$ and noise $\left[F_{(1,28)}=5.93, p=0.022\right]$ on A-P sway, a main effect of noise $\left[F_{(1,28)}=\right.$ 8.86, $p=0.006$ ) on M-L sway, and main effects of vision $[F(1,28)=10.47, p=$ 0.003]and noise $\left[\mathrm{F}_{(1,28)}=9.01, p=\right.$ $0.006]$ on radial sway."

0.006 ] on radia sway."

(A) maintain posture, "The detailed analysis using the TUG and to speeded responses postural stability test scores in the old adults further demonstrated that the enhanced visuotactile interactions were especially prevalent among the older adults with relatively poor TUG and postural stability per formance."

(A) respond to all stimuli "Maximal unipedal stance time was by pressing a stationary highest for superior and good integrators foot pedal, (B) Maintain (16.43 and 16.83s) and lowest for poor balance and deficient integrators (13.49 and $12.57 \mathrm{~s})$. Results from the linear regression analyses reveal that vs. integration is associated with maximum unipedal stance time $(\beta=0.15, p \leq 0.013) . "$

(A) Stable surface with eyes open or Maintain posture closed, (B) compliant surface with eyes open or closed on

dults with an average age of 85 years, the control of lateral sway in both quiet standing and a postural tracking task was found to be related to timed chair standing performance and cognitive processing speed, respectively."

(A) Floor with eyes open, (B) floor with (A) Maintain balance, "Overall, sway area increased significantly eyes closed, (C) foam cushion with $\quad$ (B) perceived postural eyes open (D) foam cushion with eyes stability closed

conditions progressively more difficult, and all pairwise comparisons were significant (p's $<0.004)$."

(A) Postural task: (1) Visual conditions: (A) Maintain posture, (a) fixed visual scene, (b) Eyes closed, (B) (1)Press a button (c) Sway-referenced visual scene; (2) the side an arrow Platform conditions: (a) Fixed pointed; (2) Press the platform, (b) Sway-referenced platform; (B) Cogni toward the arrow Perceptual task: (a) Congruent side pointed or on the side arrow pointed and position arrow, (b) opposite the arrow Incongruent; (2) Motor task pointed

"The EQ scores varied across the SOT There were significant correlations for the four cognitive measures within the SOT conditions." 
TABLE 5 | Studies grouped in terms of tests used.

\begin{tabular}{|c|c|c|c|}
\hline Tests & Studies & Measurements & Differences \\
\hline \multirow[t]{3}{*}{ RT paradigm } & Mahoney et al. (2014) & Reaction time & Uni-pedal stance time in different multisensory integration \\
\hline & Teramoto et al. (2017) & & $\begin{array}{l}\text { Average reaction time based on the TUG scores or } \\
\text { postural stability test scores }\end{array}$ \\
\hline & Mahoney et al. (2018) & & Uni-pedal stance time in different multisensory integration \\
\hline Sound-induced flash illusion & $\begin{array}{l}\text { Setti et al. (2011), Stapleton } \\
\text { et al. (2014) }\end{array}$ & $\begin{array}{l}\text { Percentage of correct } \\
\text { responses }\end{array}$ & - \\
\hline \multirow[t]{3}{*}{ Sensory reweighting } & Allison et al. (2006) & Gain and phase & $\begin{array}{l}\text { Moving visual stimuli in a moving room and touching a } \\
\text { moving touch plate }\end{array}$ \\
\hline & Jeka et al. (2006) & & Visual display oscillating at different amplitudes \\
\hline & Jeka et al. (2010) & & \\
\hline \multirow{6}{*}{$\begin{array}{l}\text { Modified clinical test of sensory } \\
\text { integration for balance }\end{array}$} & Rosengren et al. (2007) & Equilibrium scores & Different measurement \\
\hline & Zhou et al. (2013) & $\begin{array}{l}\text { Functional gait assessment } \\
\text { scores }\end{array}$ & Different measurement \\
\hline & Palazzo et al. (2015) & Center of pressure & Various surfaces with eyes open or closed \\
\hline & Ross et al. (2016) & & $\begin{array}{l}\text { A force platform with eyes open or closed under quiet or } \\
\text { noisy condition }\end{array}$ \\
\hline & Sparto et al. (2018) & & Various surfaces with eyes open or closed \\
\hline & Anson et al. (2019) & Center of mass sway area & Different measurement \\
\hline \multirow[t]{3}{*}{ Inhibitory function testing } & Redfern et al. (2001) & Inhibitory measures & With eyes open or closed \\
\hline & Redfern et al. (2009) & & In the light or in the dark \\
\hline & Redfern et al. (2019) & & With eyes open or closed \\
\hline
\end{tabular}

on the function. Upon literature searching, we identified 17 papers on the relationship between multisensory integration and balance function in elderly people, and these studies preliminarily suggested that multisensory integration disorder leads to a decline in balance function in elderly people (Figure 2 ).

Multisensory integration disorder refers to the abnormality in the integration of different sensations or modalities when relevant factors of balance are significantly altered. Impaired information processing in an aging brain has been attributed to prefrontal cortico-cortico facilitation (Knight et al., 1999), dedifferentiation (Dustman and Snyder, 1981), and prefrontothalamo-cortical gating (Zikopoulos and Barbas, 2006). The neural mechanisms might lie in that peripheral stimuli are analyzed and processed by corresponding sensory cortices, from which afferents are directly transmitted into cortical association area. Cortical association area is one of brain areas where multisensory neurons lie. Also, multisensory neurons have recently been discovered in modality-specific areas, such as the visual cortex, and the junctional zone between cerebral lobes, such as the occipito-temporal space and the occipito-parietal space (Wallace, 2004; Wallace et al., 2004). The subcortical areas of superior colliculus (Wallace, 2004) and putamen (Graziano and Gross, 1993; Gentile et al., 2011) were also found to be involved in multisensory integration. And other researchers believe that the basic structure of sensory system involves a series of neuronal interactions between the thalamus and the neocortex that allow efficient processing of sensory and cognitive information (Sherman and Guillery, 1996; Sherman, 2005). The thalamus plays a vital role in the cortico-cortical communication and the integration of multisensory information. Therefore, damage to cortical areas, subcortical areas, and thalamus may result in the deficits of multisensory integration.

A variety of experimental paradigms have been used to investigate multisensory integration. Of them, illusions, such as ventriloquist illusion (Alais and Burr, 2004; Radeau and Bertelson, 2018), McGurk effect (McGurk and Macdonald, 1976), and sound-induced flash illusion (Shams et al., 2000) are of particular interest to researchers since they help them gain insight into the mechanisms about the management of conflicting multisensory information. The results of this review indicated that elderly people with poor stability tend to integrate or include all the information from surrounding environment while elderly people with good stability usually weigh and screen the information to achieve balance. On the basis of the systematic analysis of relevant papers, we reviewed the theories and hypotheses on how multisensory integration works on balance function in elderly people.

\section{Inverse Effectiveness}

First of all, "inverse effectiveness" theory, initially proposed by Meredith and Stein (1986), might, to some degrees, explain how multisensory integration works on balance in elderly people. "Inverse effectiveness" theory believes that the effectiveness of multisensory integration increases when the effectiveness of the best modality-specific stimulus declines (Stein and Stanford, 2008). Some studies suggested that when stimuli from different modalities are congruent, then the benefit of multisensory inputs to perception is greater in elderly than in younger adults (Laurienti et al., 2006; Peiffer et al., 2007). However, when incongruent inputs from different modalities are combined, the 


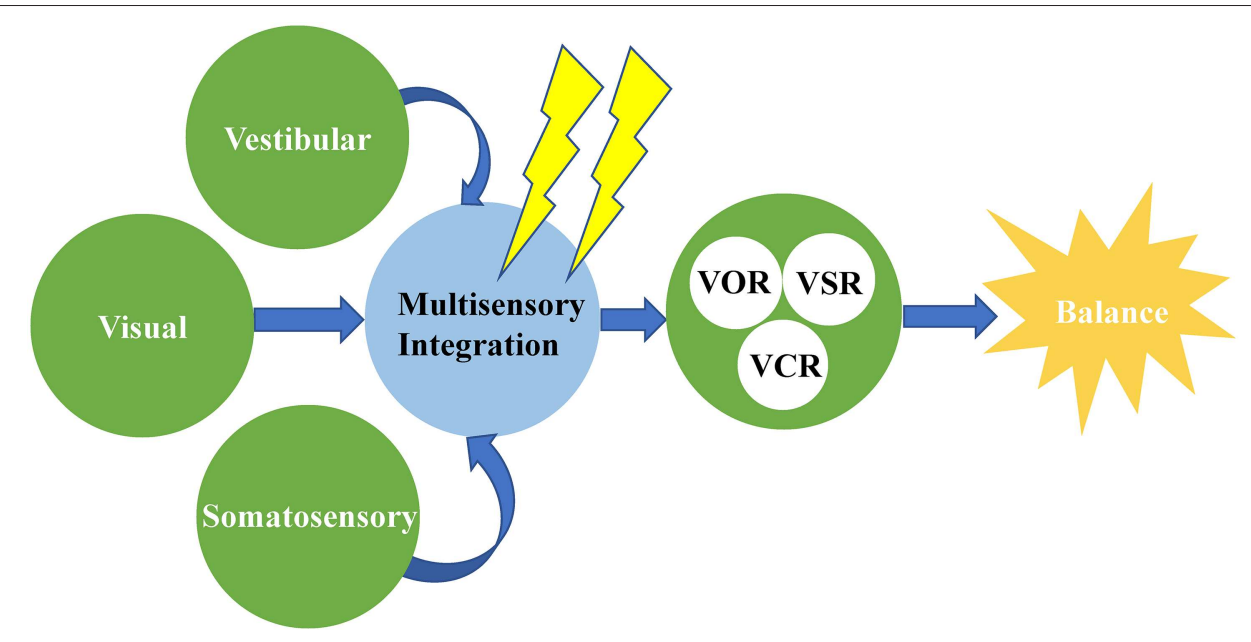

FIGURE 2 | Multisensory integration disorder leads to a decline in balance function in older adults. VOR, vestibulo-ocular reflex; VSR, vestibulospinal reflex; VCR, vestibulocolic reflex.

combination can result in inefficient processing of the inputs in elderly people (Poliakoff et al., 2006). Given the functional deterioration of sensory system in the eldely, such increased effectiveness of multisensory integration might help the elderly avail of more information from the environment to maintain balance. In the included studies, all participants underwent an intensive clinical assessment, including sensory acuity test, and were found to have no unisensory impairment. These studies showed that even when baseline values were not significantly different, elderly people with poor balance function had less multisensory enhancement (Mahoney et al., 2014). Moreover, the difference between two groups lies in multisensory rather than unisensory processing, suggesting that the difference in the processing of information is of central instead of peripheral nature. It is possible, therefore, that the inverse effectiveness of the balance-related sensations like vision, vestibular sensation and somatic sensation in healthy elderly people is super-additive or additive while fall-prone elderly people only have a subadditive enhancement (Stein et al., 2009).

\section{Deficits in Attentional Control}

Another theory is "deficits in attentional control." The attention involves a mechanism that determines how to select sensory inputs for further processing from a series of concurrent stimuli (Talsma et al., 2010). We live in a multisensory world in which we are continuously exposed to stimuli via multiple sensory pathways. For effective cognition, we must continually select and appropriately integrate those inputs that are most relevant to our behavioral goals. Recently, multiple researches suggested that many falls in balance-impaired elderly people occurred not when they were simply walking, but when they were walking and simultaneously performing another task, such as talking (Shumway-Cook and Woollacott, 2000; Woollacott and Shumway-Cook, 2002). These studies supported the notion that attentional demand associated with postural control is higher in fall-prone elderly people. Latinus et al. (2010) believe that stimulus-driven, bottom-up mode induced by integration of multisensory inputs can automatically capture attention toward multisensory events. Conversely, with the top-down mode, attention can facilitate the integration of multisensory inputs and lead to a spread of attention across sensory modalities. Recent studies showed that multisensory bottom-up processes are conductive to the capture and selection of attention. In turn, attention can affect the effectiveness of multisensory integration in a top-down fashion. Andres et al. (2006) demonstrated that the elderly had deficits in attentional control and were more likely to be distracted by stimuli from different sensory modalities. These findings suggest that there exists a closer and multifactorial interaction between attention and multisensory integration. The dynamic and bidirectional interplay between attentional selection and multisensory processing is fundamental to postural control and balance maintenance. Therefore, when the elderly try to maintain balance and simultaneously perform another task, the activity of the brain region associated with balance is significantly reduced while the brain areas of unrelated modalities are, on the contrary, more active (Mozolic et al., 2012), especially in fall-prone elderly people. The beneficial effect of attentional control on balance maintenance are reduced and fall results.

\section{Larger Time Window of Integration}

Finally, another important theory concerning multisensory integration is the "larger time window of integration" hypothesis. A common finding across many studies was that elderly people had an increased response time (Diederich et al., 2008; Mozolic et al., 2012). Diederich et al. (2008) used a time-windowof-integration (TWIN) model to distinguish between the relative contributions of early peripheral sensory processes and subsequent central integration to multisensory enhancement. They found that the larger time window of integration in the elderly is primarily the result of slower and more variable peripheral sensory processing. Sound-induced flash illusion is 
one of the most common phenomena resulting from larger time window of integration. When auditory and visual stimuli are presented rapidly, the number of auditory stimuli can affect the number of visual stimuli perceived (Bizley et al., 2016). Studies on the sound-induced flash illusion showed that the integrated time window was larger in fall-prone elderly people (Setti et al., 2011; Stapleton et al., 2014). Bloem et al. (2006) found evidence for a "posture-first" strategy, by which participants performing dualtasks sacrificed performance on perceptual tasks and prioritized balance control. Therefore, the fall-prone elderly people have less allocation of attentional resources to the multisensory task when maintaining balance. Such insufficient allocation leads to an increase in faulty percepts when multisensory information is incongruent (Stapleton et al., 2014). But how exactly the larger time window of integration affects balance is still unknown. Setti et al. (2011) put forward a speculation that either an indirect or a direct effect might be involved in the mechanism. On the one hand, balance can be indirectly challenged by the processing of irrelevant sensory information due to the larger time window. On the other hand, fall-prone elderly people tend to over-depend on multisensory stimulation. Therefore, when exposed to incongruent stimuli, fall-prone elderly people have a specific difficulty in processing the information. In turn, the larger temporal window may exert a direct effect on their ability to maintain balance.

Some other theories should also be mentioned here. It has been shown that the brain of older adults tends to have less asymmetric hemispheric activation, a phenomenon called HAROLD (hemispheric asymmetry reduction in older adults) (Cabeza, 2002). And it states that, during multisensory tasks, the elderly recruit more brain areas. HAROLD was found to be correlated with higher performance in task execution in the elderly, which prompts a hypothesis that these changes take place to preserve cognitive function in elderly people. One possible explanation is that the mechanism is a compensation for aging (Peters, 2006). However, the relationship between brain recruitment strategies and balance maintenance has not yet been fully understood. Another explanation concerns the increased noise at baseline (Mozolic et al., 2012). When elderly people are engaged in selective attention, multisensory enhancement in their brain remains and can benefit them when all information is reliable. However, when some irrelevant information is present, this increased baseline becomes a disadvantage.

The major finding of the study was that appropriate integration of information from multisensory modalities is essential for elderly people to maintain balance. The hypothesis of deficits in attentional control and larger time window of integration can better explain why the multisensory integration impairment is associated with impaired balance function in elderly people.

\section{Strengths and Limitations}

Our review has several strengths. This systematic review was based on the guidelines of the PRISMA statement (Moher et al., 2009), a tool designed to enhance the quality of systematic reviews. All accessible databases were searched, including WOS,
PubMed, Scopus, among others, which maximized the number of eligible studies. What's more, the quality of case control studies was assessed against NOS (Wells et al., 2012). Only high-quality studies were included in qualitative synthesis. The quality of cross-sectional studies was assessed using an 11-item checklist which was recommended by AHRQ. Most importantly, our systematic review found that elderly people with poor stability tend to integrate or include all the information from surrounding environment while elderly people with good stability usually weigh and screen the information to achieve balance. Moreover, multisensory integration might work on balance function in elderly people.

This study had some limitations. The eligible studies yielded different results concerning the effect of multisensory integration, so it was impossible to conduct a meta-analysis. And the different methods used in the 17 included studies also made it difficult to analyze the overall effect of the multisensory integration on balance function. Furthermore, we only reviewed the abstracts and full texts that were published in English and Chinese. Relevant papers in other languages might be not included. And some relevant studies might be missed if authors didn't list their studies as being related to multisensory integration.

\section{CONCLUSIONS}

In conclusion, this systematic review looked into how multisensory integration works on balance function in elderly people and found that the impairment of multisensory integration will predispose elderly people to fall. Accurate assessment of multisensory integration can help the elderly identify the impairment of balance function and minimize the risk of fall. And our results provide a new basis for further understanding of mechanisms of balance maintenance. Further research is warranted to explore the changes in brain areas related to multisensory integration in elderly people.

\section{DATA AVAILABILITY STATEMENT}

The raw data supporting the conclusions of this article will be made available by the authors, without undue reservation, to any qualified researcher.

\section{AUTHOR CONTRIBUTIONS}

SZ and WX: study conception, design, data extraction, data analysis and interpretation, and drafting and revision of the manuscript. YZ and ET: data extraction and critical revision. WK: study design and critical revision. All authors approved the manuscript.

\section{FUNDING}

This study was supported by grants from the National TwelfthFive Year Research Program of China (No. 2012BAI12B02) and the National Natural Science Foundation of China (No. 81873701). 


\section{REFERENCES}

Alais, D., and Burr, D. (2004). The ventriloquist effect results from near-optimal bimodal integration. Curr. Biol. 14, 257-262. doi: 10.1016/j.cub.2004.01.029

Allison, L. K., Kiemel, T., and Jeka, J. J. (2006). Multisensory reweighting of vision and touch is intact in healthy and fall-prone older adults. Exp. Brain Res. 175, 342-352. doi: 10.1007/s00221-006-0559-7

Andres, P., Parmentier, F. B., and Escera, C. (2006). The effect of age on involuntary capture of attention by irrelevant sounds: a test of the frontal hypothesis of aging. Neuropsychologia 44, 2564-2568. doi: 10.1016/j.neuropsychologia.2006.05.005

Anson, E., Studenski, S., Sparto, P. J., and Agrawal, Y. (2019). Communitydwelling adults with a history of falling report lower perceived postural stability during a foam eyes closed test than non-fallers. Exp. Brain Res. 237, 769-776. doi: 10.1007/s00221-018-5458-1

Barutchu, A., Sahu, A., Humphreys, G. W., and Spence, C. (2019). Multisensory processing in event-based prospective memory. Acta Psychol. 192, 23-30. doi: 10.1016/j.actpsy.2018.10.015

Bizley, J. K., Maddox, R. K., and Lee, A. K. C. (2016). Defining auditory-visual objects: behavioral tests and physiological mechanisms. Trends Neurosci. 39, 74-85. doi: 10.1016/j.tins.2015.12.007

Blake, A. J., Morgan, K., Bendall, M. J., Dallosso, H., Ebrahim, S. B., Arie, T. H., et al. (1988). Falls by elderly people at home: prevalence and associated factors. Age Ageing 17, 365-372. doi: 10.1093/ageing/17.6.365

Bloem, B.R., Grimbergen, Y.A., van Dijk, J.G., and Munneke, M. (2006). The "posture second" strategy: a review of wrong priorities in Parkinson's disease. J. Neurol. Sci. 248:196-204. doi: 10.1016/j.jns.2006.05.010

Bolognini, N., Russo, C., and Vallar, G. (2015). Crossmodal illusions in neurorehabilitation. Front. Behav. Neurosci. 9:212. doi: 10.3389/fnbeh.2015.00212

Bronstein, A. M. (2016). Multisensory integration in balance control. Handb. Clin. Neurol. 137, 57-66. doi: 10.1016/B978-0-444-63437-5.00004-2

Cabeza, R. (2002). Hemispheric asymmetry reduction in older adults: the HAROLD model. Psychol. Aging 17, 85-100. doi: 10.1037/0882-7974.17.1.85

Chisolm, T. H., Willott, J. F., and Lister, J. J. (2003). The aging auditory system: anatomic and physiologic changes and implications for rehabilitation. Int. J. Audiol. 42, 3-10. doi: 10.3109/14992020309074637

de Dieuleveult, A. L., Siemonsma, P. C., van Erp, J. B. F., and Brouwer, A.-M. (2017). Effects of aging in multisensory integration: a systematic review. Front. Aging Neurosci. 9:80 doi: 10.3389/fnagi.2017.00080

DeLoss, D. J., Pierce, R. S., and Andersen, G. J. (2013). Multisensory integration, aging, and the sound-induced flash illusion. Psychol. Aging 28, 802-812. doi: $10.1037 / \mathrm{a} 0033289$

Diaconescu, A. O., Hasher, L., and McIntosh, A. R. (2013). Visual dominance and multisensory integration changes with age. Neuroimage 65, 152-166. doi: 10.1016/j.neuroimage.2012.09.057

Diederich, A., Colonius, H., and Schomburg, A. (2008). Assessing age-related multisensory enhancement with the time-window-of-integration model. Neuropsychologia 46, 2556-2562. doi: 10.1016/j.neuropsychologia.2008.03.026

Dustman, R. E., and Snyder, E. W. (1981). Life-span changes in visually evoked potentials at central scalp. Neurobiol. Aging 2, 303-308. doi: 10.1016/0197-4580(81)90039-7

Foxe, J. J., Morocz, I. A., Murray, M. M., Higgins, B. A., Javitt, D. C., and Schroeder, C. E. (2000). Multisensory auditory-somatosensory interactions in early cortical processing revealed by high-density electrical mapping. Cogn. Brain Res. 10, 77-83. doi: 10.1016/S0926-6410(00)00024-0

Gentile, G., Petkova, V. I., and Ehrsson, H. H. (2011). Integration of visual and tactile signals from the hand in the human brain: an FMRI study. $J$. Neurophysiol. 105, 910-922. doi: 10.1152/jn.00840.2010

Graziano, M. S., and Gross, C. G. (1993). A bimodal map of space: somatosensory receptive fields in the macaque putamen with corresponding visual receptive fields. Exp. Brain Res. 97, 96-109. doi: 10.1007/BF002 28820

Jeka, J., Allison, L., Saffer, M., Zhang, Y., Carver, S., and Kiemel, T. (2006). Sensory reweighting with translational visual stimuli in young and elderly adults: the role of state-dependent noise. Exp. Brain Res. 174, 517-527. doi: 10.1007/s00221-006-0502-y
Jeka, J. J., Allison, L. K., and Kiemel, T. (2010). The dynamics of visual reweighting in healthy and fall-prone older adults. J. Mot. Behav. 42, 197-208. doi: 10.1080/00222895.2010.481693

Katsarkas, A. (1994). Dizziness in aging: a retrospective study of 1194 cases. Otolaryngol. Head Neck Surg. 110, 296-301. doi: 10.1177/019459989411000306

Kinchla, R. A. (1974). Detecting target elements in multielement arrays: a confusability model. Percept. Psychophys. 15, 149-158. doi: $10.3758 / \mathrm{BF} 03205843$

Knight, R. T., Richard Staines, W., Swick, D., and Chao, L. L. (1999). Prefrontal cortex regulates inhibition and excitation in distributed neural networks. Acta Psychol. 101, 159-178. doi: 10.1016/S0001-6918(99)00004-9

Latinus, M., VanRullen, R., and Taylor, M. J. (2010). Top-down and bottom-up modulation in processing bimodal face/voice stimuli. BMC Neurosci. 11, 36-36. doi: 10.1186/1471-2202-11-36

Laurienti, P. J., Burdette, J. H., Maldjian, J. A., and Wallace, M. T. (2006). Enhanced multisensory integration in older adults. Neurobiol. Aging 27, 1155-1163. doi: 10.1016/j.neurobiolaging.2005.05.024

Lowry, K. A., Vallejo, A. N., and Studenski, S. A. (2012). Successful aging as a continuum of functional independence: lessons from physical disability models of aging. Aging Dis. 3, 5-15.

Ma, H., Sun, G., Wang, W., Zhou, Y., Liu, D., Tong, Y., et al. (2016). Association between Interleukin-6-572 C $>\mathrm{G}$ and $-174 \mathrm{G}>\mathrm{C}$ polymorphisms and hypertension. Medicine 95:e2416. doi: 10.1097/MD.0000000000002416

Mahoney, J. R., Cotton, K., and Verghese, J. (2018). Multisensory integration predicts balance and falls in older adults. J. Gerontol. 74, 1429-1435. doi: 10.1093/gerona/gly245

Mahoney, J. R., Holtzer, R., and Verghese, J. (2014). Visual-somatosensory integration and balance: evidence for psychophysical integrative differences in aging. Multisens. Res. 27, 17-42. doi: 10.1163/22134808-00002444

McGurk, H., and Macdonald, J. (1976). Hearing lips and seeing voices. Nature 264, 746-748. doi: 10.1038/264746a0

Meredith, M. A., and Stein, B. E. (1986). Visual, auditory, and somatosensory convergence on cells in superior colliculus results in multisensory integration. J. Neurophysiol. 56, 640-662. doi: 10.1152/jn.1986.56.3.640

Moher, D., Liberati, A., Tetzlaff, J., and Altman, D. G. (2009). Preferred reporting items for systematic reviews and meta-analyses: the PRISMA statement. PLoS Med. 6:e1000097. doi: 10.1371/journal.pmed.1000097

Morís Fernández, L., Macaluso, E., and Soto-Faraco, S. (2017). Audiovisual integration as conflict resolution: the conflict of the McGurk illusion. Hum. Brain Mapp. 38, 5691-5705. doi: 10.1002/hbm.23758

Mozolic, J. L., Hugenschmidt, C. E., Peiffer, A. M., and Laurienti, P. J. (2012). "Multisensory integration and aging," in The Neural Bases of Multisensory Processes, eds. M. M. Murray and M. T. Wallace (Boca Raton, FL: CRC Press/Taylor \& Francis Llc.), 81-392. doi: 10.1201/9781439812174-25

Palazzo, F., Caronti, A., Lebone, P., Proietti, A., Panzarino, M., and Annino, G. (2015). Effects of stimulating surface during static upright posture in the elderly. Somatosensory Motor Res. 32, 61-66. doi: $10.3109 / 08990220.2014 .958217$

Peelle, J. E., and Sommers, M. S. (2015). Prediction and constraint in audiovisual speech perception. Cortex 68, 169-181. doi: 10.1016/j.cortex.2015.03.006

Peiffer, A. M., Mozolic, J. L., Hugenschmidt, C. E., and Laurienti, P. J. (2007). Age-related multisensory enhancement in a simple audiovisual detection task. Neuroreport 18, 1077-1081. doi: 10.1097/WNR.0b013e3281e72ae7

Peters, R. (2006). Ageing and the brain. Postgrad. Med. J. 82, 84-88. doi: 10.1136/pgmj.2005.036665

Poliakoff, E., Ashworth, S., Lowe, C., and Spence, C. (2006). Vision and touch in ageing: crossmodal selective attention and visuotactile spatial interactions. Neuropsychologia 44, 507-517. doi: 10.1016/j.neuropsychologia.2005.07.004

Price, D. D. (1999). Multisensory integration in pain and consciousness. Pain Forum 8, 130-132. doi: 10.1016/S1082-3174(99)70022-X

Radeau, M., and Bertelson, P. (2018). The After-Effects of Ventriloquism. Quart. J. Exp. Psychol. 26, 63-71. doi: 10.1080/14640747408400388

Ramkhalawansingh, R., Keshavarz, B., Haycock, B., Shahab, S., and Campos, J. L. (2016). Age differences in visual-auditory self-motion perception during a simulated driving task. Front. Psychol. 7:595 doi: 10.3389/fpsyg.2016.00595

Redfern, M. S., Chambers, A. J., Sparto, P. J., Furman, J. M., and Jennings, J. R. (2019). Inhibition and decision-processing speed are associated with 
performance on dynamic posturography in older adults. Exp. Brain Res. 237, 37-45. doi: 10.1007/s00221-018-5394-0

Redfern, M. S., Jennings, J. R., Martin, C., and Furman, J. M. (2001). Attention influences sensory integration for postural control in older adults. Gait Posture 14, 211-216. doi: 10.1016/S0966-6362(01)00144-8

Redfern, M. S., Jennings, J. R., Mendelson, D., and Nebes, R. D. (2009). Perceptual inhibition is associated with sensory integration in standing postural control among older adults. J. Gerontol. B Psychol. Sci. Soc. Sci. 64, 569-576. doi: 10.1093/geronb/gbp060

Rosengren, K. S., Rajendran, K., Contakos, J., Chuang, L.-L., Peterson, M., Doyle, R., et al. (2007). Changing control strategies during standard assessment using computerized dynamic posturography with older women. Gait Posture 25, 215-221. doi: 10.1016/j.gaitpost.2006.03.009

Ross, J. M., Will, O. J., McGann, Z., and Balasubramaniam, R. (2016). Auditory white noise reduces age-related fluctuations in balance. Neurosci. Lett. 630, 216-221. doi: 10.1016/j.neulet.2016.07.060

Rubenstein, L. Z. (2006). Falls in older people: epidemiology, risk factors and strategies for prevention. Age Ageing. 35(suppl. 2), ii37-ii41. doi: 10.1093/ageing/afl084

Schroeder, C. E., and Foxe, J. (2005). Multisensory contributions to lowlevel, 'unisensory' processing. Curr. Opin. Neurobiol. 15, 454-458. doi: 10.1016/j.conb.2005.06.008

Seitz, A. R., Kim, R., and Shams, L. (2006). Sound facilitates visual learning. Curr. Biol. 16, 1422-1427. doi: 10.1016/j.cub.2006.05.048

Setti, A., Burke, K. E., Kenny, R. A., and Newell, F. N. (2011). Is inefficient multisensory processing associated with falls in older people? Exp. Brain Res. 209, 375-384. doi: 10.1007/s00221-011-2560-Z

Shams, L., Kamitani, Y., and Shimojo, S. (2000). Illusions - What you see is what you hear. Nature 408, 788-788. doi: 10.1038/35048669

Sherman, S. M. (2005). Thalamic relays and cortical functioning. Prog. Brain Res. 149, 107-126. doi: 10.1016/S0079-6123(05)49009-3

Sherman, S. M., and Guillery, R. W. (1996). Functional organization of thalamocortical relays. J. Neurophysiol. 76, 1367-1395. doi: 10.1152/jn.1996.76.3.1367

Shumway-Cook, A., and Woollacott, M. (2000). Attentional demands and postural control: the effect of sensory context. J. Gerontol. A Biol. Sci. Med. Sci. 55:M1016. doi: 10.1093/gerona/55.1.m10

Sparto, P. J., Newman, A. B., Simonsick, E. M., Caserotti, P., Strotmeyer, E. S., Kritchevsky, S. B., et al. (2018). Contributions to lateral balance control in ambulatory older adults. Aging Clin. Exp. Res. 30, 633-641. doi: 10.1007/s40520-017-0819-3

Spence, C. (2011). Crossmodal correspondences: a tutorial review. Attent. Percept. Psychophys. 73, 971-995. doi: 10.3758/s13414-010-0073-7

Stalenhoef, P. A., Diederiks, J. P., Knottnerus, J. A., Kester, A. D., and Crebolder, H. F. (2002). A risk model for the prediction of recurrent falls in communitydwelling elderly: a prospective cohort study. J. Clin. Epidemiol. 55, 1088-1094. doi: 10.1016/S0895-4356(02)00502-4

Stapleton, J., Setti, A., Doheny, E. P., Kenny, R. A., and Newell, F. N. (2014). A standing posture is associated with increased susceptibility to the soundinduced flash illusion in fall-prone older adults. Exp. Brain Res. 232, 423-434. doi: 10.1007/s00221-013-3750-7

Stein, B. E., and Stanford, T. R. (2008). Multisensory integration: current issues from the perspective of the single neuron. Nat. Rev. Neurosci. 9, 255-266. doi: $10.1038 / \mathrm{nrn} 2331$

Stein, B. E., Stanford, T. R., Ramachandran, R., Perrault, T. J., and Rowland, B. A. (2009). Challenges in quantifying multisensory integration: alternative criteria, models, and inverse effectiveness. Exp. Brain Res. 198, 113-126. doi: 10.1007/s00221-009-1880-8

Stel, V. S., Smit, J. H., Pluijm, S. M. F., and Lips, P. (2003). Balance and mobility performance as treatable risk factors for recurrent falling in older persons. $J$. Clin. Epidemiol. 56, 659-668. doi: 10.1016/S0895-4356(03)00082-9
Stephen, J. M., Knoefel, J. E., Adair, J., Hart, B., and Aine, C. J. (2010). Aging-related changes in auditory and visual integration measured with MEG. Neurosci. Lett. 484, 76-80. doi: 10.1016/j.neulet.2010.08.023

Talsma, D. (2015). Predictive coding and multisensory integration: an attentional account of the multisensory mind. Front. Integr. Neurosci. 9:19. doi: 10.3389/fnint.2015.00019

Talsma, D., Senkowski, D., Soto-Faraco, S., and Woldorff, M. G. (2010). The multifaceted interplay between attention and multisensory integration. Trends Cogn. Sci. 14, 400-410. doi: 10.1016/j.tics.2010.06.008

Teasdale, N., Stelmach, G. E., Breunig, A., and Meeuwsen, H. J. (1991). Age differences in visual sensory integration. Exp. Brain Res. 85, 691-696. doi: 10.1007/BF00231755

Teramoto, W., Honda, K., Furuta, K., and Sekiyama, K. (2017). Visuotactile interaction even in far sagittal space in older adults with decreased gait and balance functions. Exp. Brain Res. 235, 2391-2405. doi: 10.1007/s00221-017-4975-7

Tromp, A. M., Pluijm, S. M., Smit, J. H., Deeg, D. J., Bouter, L. M., and Lips, P. (2001). Fall-risk screening test: a prospective study on predictors for falls in community-dwelling elderly. J. Clin. Epidemiol. 54, 837-844. doi: 10.1016/S0895-4356(01)00349-3

United Nations (2017). “World Population Ageing 2017”. New York, NY: United Nations. doi: 10.18356/88fa44e7-en

Wallace, M. T. (2004). The development of multisensory processes. Cogn. Process. 5, 69-83. doi: 10.1007/s10339-004-0017-z

Wallace, M. T., Ramachandran, R., and Stein, B. E. (2004). A revised view of sensory cortical parcellation. Proc. Natl. Acad. Sci. U.S.A. 101, 2167-2172. doi: 10.1073/pnas.0305697101

Wassenhove, V. V., Grant, K. W., Poeppel, D., and Halle, M. (2005). Visual speech speeds up the neural processing of auditory speech. Proc. Natl. Acad. Sci. U.S.A. 102, 1181-1186. doi: 10.1073/pnas.0408949102

Wells, G., Shea, B., O'Connell, D., Peterson, J., Welch, V., Losos, M., et al. (2012). The Newcastle-Ottawa Scale (NOS) for Assessing the Quality of Nonrandomised Studies in Meta-Analyses. Available online at: http://www.ohri.ca/programs/ clinical_epidemiology/oxford.asp

Woollacott, M., and Shumway-Cook, A. (2002). Attention and the control of posture and gait: a review of an emerging area of research. Gait \& Posture, 16:1-14. doi: 10.1016/s0966-6362(01)00156-4

World Health Organization (2007). Global Report on Falls Prevention in Older People. Geneva: World Health Organization.

World Health Organization (2015). World Report on Ageing and Health. Geneva: World Health Organization.

Zhou, M., Peng, N., Shi, R., Fang, J., Li, L., Li, H., et al. (2013). Physical function tests for predicting falls among community-dwelling older adults. Chinese J. Phys. Med. Rehabil. 35, 456-459. doi: 10.3760/cma.j.issn.0254-1424.2013. 06.006

Zikopoulos, B., and Barbas, H. (2006). Prefrontal projections to the thalamic reticular nucleus form a unique circuit for attentional mechanisms. J. Neurosci. 26, 7348-7361. doi: 10.1523/JNEUROSCI.551105.2006

Conflict of Interest: The authors declare that the research was conducted in the absence of any commercial or financial relationships that could be construed as a potential conflict of interest.

Copyright (c) 2020 Zhang, Xu, Zhu, Tian and Kong. This is an open-access article distributed under the terms of the Creative Commons Attribution License (CC BY). The use, distribution or reproduction in other forums is permitted, provided the original author(s) and the copyright owner(s) are credited and that the original publication in this journal is cited, in accordance with accepted academic practice. No use, distribution or reproduction is permitted which does not comply with these terms. 